\title{
EL SERVICIO EXTERIOR ESPAÑOL: UNA VISIÓN CRÍTICA DESDE SU CREACIÓN HASTA LA REFORMA DE 2006
}

\author{
Alberto Priego 1 \\ UNISCI/ Universidad Pontificia Comillas
}

\begin{abstract}
Resumen:
La Sociedad Internacional está sufriendo importantes transformaciones desde el fin de la Guerra Fría. Los servicios diplomáticos están tratando de adaptarse a dichos cambios. Así, el Foreign Service, el Foreign Office entre otros han emprendido diferentes procesos de reforma. El Servicio Exterior Español inició este mismo proceso en 2005. Este artículo trata de analizar cómo se ha acometido dicha transformación.
\end{abstract}

Palabras clave: Diplomacia, reforma, Ministerio de Asuntos Exteriores.

Title in English: "The Spanish Foreign Service: A Critical Assessment regarding its Creation and Reform in 2006”.

Abstract:

International Society has suffered important changes since the end of the Colf War. Because of that, the different diplomatic services, such as the Foreign Service of the Foreign Office, are trying to adapt to the new situation. In 2005, the Spanish Foreign Service (Servicio Exterior Español) started a similar transformation process. This article tries to make an assessment on this transformation.

Keywords: Diplomacy, Reform, Ministry of Foreign Affairs.

Copyright @ UNISCI, 2011.

Las opiniones expresadas en estos artículos son propias de sus autores, y no reflejan necesariamente la opinión de UNISCI. The views expressed in these articles are those of the authors, and do not necessarily reflect the views of UNISCI.

\footnotetext{
${ }^{1}$ Alberto Priego Moreno es Profesor de la Universidad Pontificia Comillas e Investigador Senior UNISCI. Email: apriego@upcomillas.es. 


\section{Introducción}

Desde hace años, el Estado está trabajando en la internacionalización de España debido a que buena parte de nuestro futuro económico depende esto. Junto a esta cuestión existen una serie de aspectos que requieren que nuestro país busque una mayor presencia a nivel internacional como puede ser la propia globalización, los retos globales o la difuminación entre las fronteras de la seguridad interior y exterior. Estos hechos nos han llevado a trabajar en un plan de internacionalización que tuvo una primera fase a comienzos del siglo XXI en la que se crearon instituciones como Casa Asia, América, África, la Secretaria de Estado para la UE o el propio Instituto Cervantes. Esta primera fase que bien podría basarse en la denominada Nueva Diplomacia requería completarla con una segunda fase enmarcada dentro de la Vieja Diplomacia y por lo tanto fomento del Sector de Acción Exterior.

\section{2. ¿Qué entendemos por diplomacia?}

Antes de comenzar este trabajo conviene hacer una serie de aclaraciones relativas al concepto mismo de diplomacia. Siguiendo al profesor Eduardo Vilariño podíamos de que diplomacia es:

"Todo Conjunto de relaciones de diversa índole que rebasan las fronteras territoriales o los ámbitos competenciales de los entes jurídicos-internacionales constituyendo un entramado de relaciones"

De esta definición se pueden obtener algunas conclusiones tales como que las relaciones diplomáticas se enmarcan dentro las relaciones internacionales o al menos transnacionales. También podemos extraer que si bien no son exclusivamente estatales si que mantiene un carácter claramente estatocéntrico y que forman un entramado de relaciones de gran complejidad. Sin embargo, alguno de los grandes teóricos de la diplomacia no consideran que el estado sea el único actor. Por ejemplo, Adam Watson afirma que la diplomacia es " $a$ negotiation of political entities which acknowledge each other's independence" Así, debemos destacar que no solo los Estados son susceptibles de desarrollar diplomacia aunque sí son los principales actors en este campo. Un claro ejemplo son las organizaciones internacionales tales como la OTAN o la UE que poseen importantes departamentos de Public Diplomacy.

Por otro lado, cabe destacar que política exterior y diplomacia no son conceptos equiparables. De hecho, la diplomacia está al servicio de la política exterior ya que los órganos centrales ${ }^{3}$ tratan de formular las líneas generales que marcarán el rumbo del Estado en el exterior y será, precisamente mediante la diplomacia quien ejecute dicha política exterior ${ }^{4}$. En principio, los órganos externos ${ }^{5}$ no participan en la formulación de la política

\footnotetext{
${ }^{2}$ Watson, Adam (1991): Diplomacy. The Dialogue between the states, London, Taylor and Francis, p. 33.

${ }^{3}$ Según el artículo 7.2. de la Convención de Viena sobre Derecho de Tratados se entiende por órganos centrales “a) los J.Estado, Jefes de Gobierno y Ministros de AAEE para la ejecución de todos los actos relativos a la celebración de tratados" Además en el caso de España tanto el art 5.a del Decreto 801/1972 de 24 de marzo como los artículos 97 y 98.2 de la Constitución habilitan al Gobierno y a su presidente a desarrollar actividades de diplomacia directa.

${ }^{4}$ Berridge, G.R (2010): Diplomacy. Theory and Practice. London, Palgrave-MacMillan p. 10

${ }^{5}$ El art 2 del Real Decreto 632/1987 dice: "La Administración del Estado en el exterior se articula en:

a)Misiones diplomáticas para el desarrollo de las relaciones diplomáticas bilaterales.

b)Representaciones Permanentes y Delegaciones para el desarrollo de las relaciones diplomáticas multilaterales"
} 
exterior, pero sí que son un importante elemento a la hora dar la información necesaria para la reformulación de la misma. De hecho la función de la información sigue siendo una de las funciones fundamentales de la diplomacia tal y como reconoce la propia Convención de Viena sobre relaciones Diplomáticas de $1961^{6}$. Algunos diplomáticos ${ }^{7}$ han instado a superar esta barrera y busca entrar en la propia formulación de la política exterior. Más allá de cambiar el sentido tradicional de la diplomacia -ser el soberano allí donde éste no pueda llegar- se estaría cometiendo un "fraude democrático" ya que el diplomático al igual que el militar no debe si no ejecutar lo que otros que han sido elegidos por el pueblo. De hecho, si los mandatarios toman decisiones equivocadas tendrán que rendir cuenta a los electores en las próximas elecciones. Por el contrario, tanto el diplomático como el militar sólo tendrán que rendir cuenta ante su "superior" y no ante el pueblo. gráfico:

Podíamos representar la relación entre diplomacia y política exterior en el siguiente

\footnotetext{
6"Art.3.1. Las funciones de una misión diplomática consisten principalmente en: d) enterarse por todos los medios lícitos de las condiciones y de la evolución de los acontecimientos en el Estado receptor e informar sobre ello al gobierno del Estado acreditante" Convención de Viena sobre Relaciones Diplomáticas 1961. Disponible en www.oas.org/legal/spanish/.../convencionviena.htm.

7 "Para desempeñarla deberán ser más abiertos y estar dispuestos a salirse de las instrucciones recibidas y entrar en un diálogo y debate auténticos" Riordan, Shaun: "La nueva Diplomacia", Foreign Policy, no. 7 (FebreroMarzo 2005), p. 5.
} 
GRÁFICO 1: Formulación y ejecución de la política exterior.
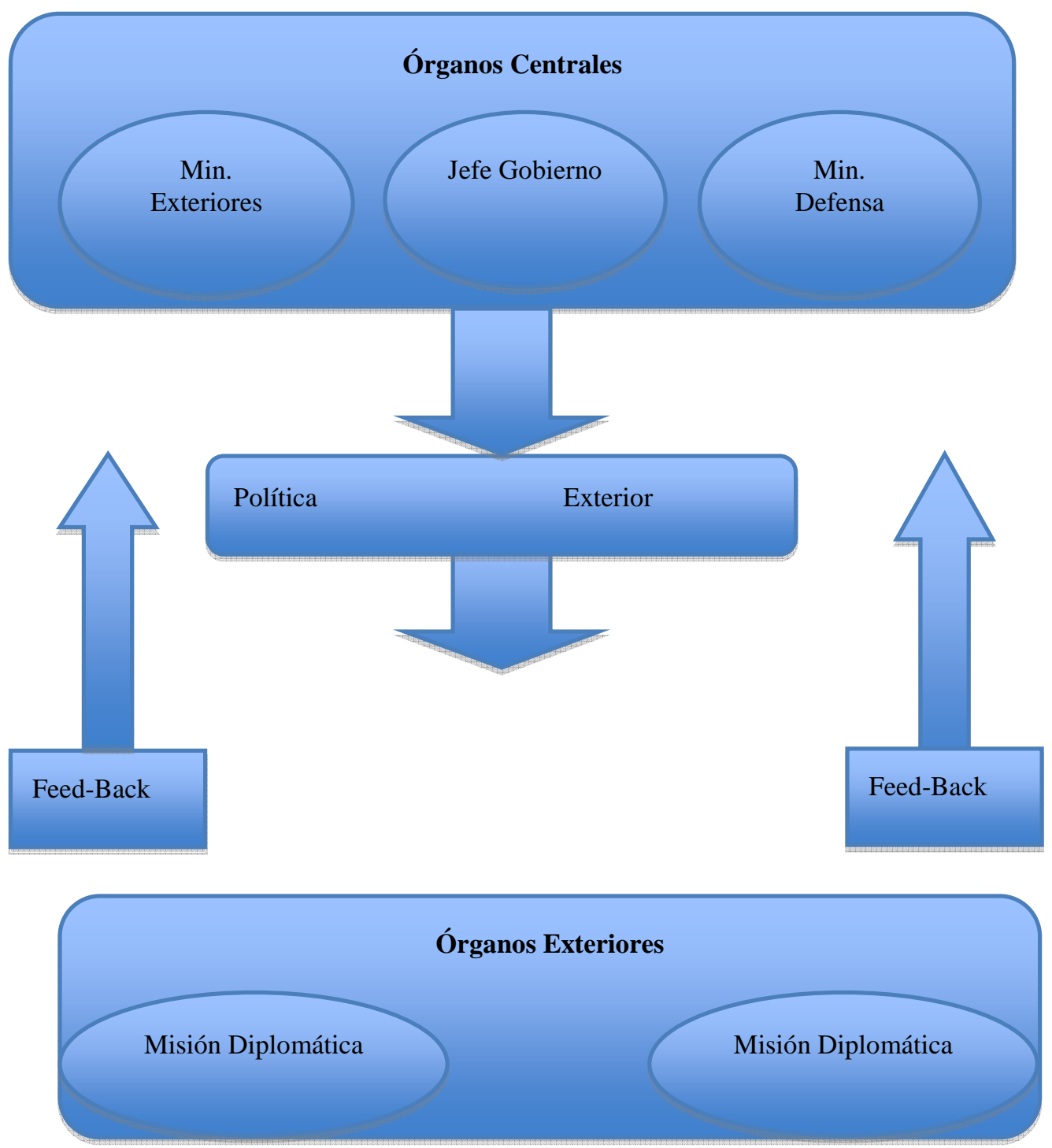

FUENTE: Elaboración Propia

Otro elemento imprescindible para la diplomacia es su desarrollo en un entorno pacífico. La gama de actuaciones en la que se desarrollan las relaciones internacionales pueden ir desde la cooperación cuya máxima expresión es la integración al conflicto cuya máxima expresión es la guerra. Por ello, podemos decir que para que se puedan dar relaciones diplomáticas, éstas, deben desarrollarse de forma pacífica. Martínez Morcillo afirmaba que el primer agente diplomático de la historia nació cuando dos comunidades decidieron enviar a una persona para negociar pacíficamente zonas de caza ${ }^{8}$.

\footnotetext{
${ }^{8}$ Pereira, Juan Carlos (ed) (2011): La Política Exterior de España. De 1800 hasta hoy, 2a Ed., Madrid, Ariel Ciencias Sociales, p. 236.
} 
Tal y como establece Watson, la diplomacia es diálogo y negociación así que de no desarrollarse bajo condiciones de paz es imposible que se puedan existir relaciones diplomáticas. Por ello, otro elemento no sólo necesario sino imprescindible es el entorno pacífico lejos de la coerción y de las amenazas.

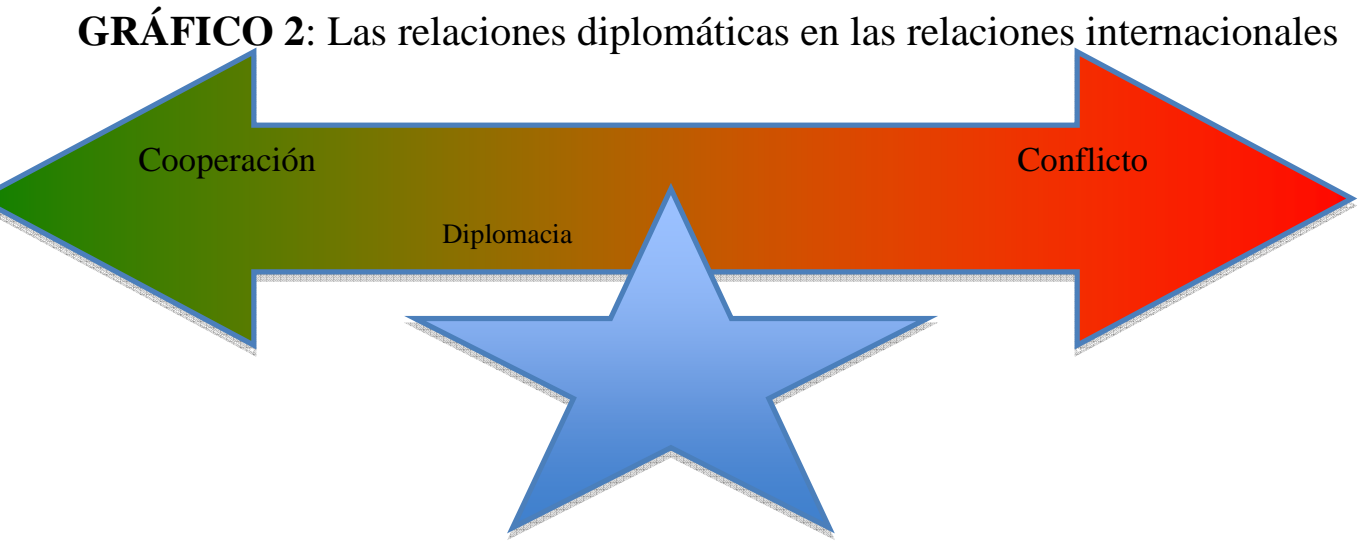

FUENTE: Elaboración Propia

Así por diplomacia podíamos entender "es aquella actividad ejecutora de la política exterior de un SDIP, al servicio de los intereses nacionales o generales de la sociedad internacional, llevada a cabo por órganos y personas debidamente representativos del mismo, ante otros $u$ otros sujetos de derecho internacional, para, por medio de la negociación, alcanzar, mantener o fortalecer transaccionalmente la paz....

Ha de tener como finalidad última hacer posible, con tales medios, no sólo el desarrollo de las relaciones pacíficas inter-subjetivas, sino, también la construcción o existencia de una sociedad internacional justa que, a través de la cooperación, permita el pleno desarrollo de los pueblos ${ }^{9,}$.

En esta definición encontramos todos los elementos que hemos venido señalando en este primer apartado:

a) Es la ejecutora de la política exterior de un Sujeto de Derecho Internacional por lo que, como órgano exterior, no participa directamente en la formulación de la política exterior.

b) Defiende unos intereses ya sean particulares en el caso de un Estado o más generales si el sujeto al que representa es una organización internacional de carácter universal. El más claro ejemplo de este aspecto son los diplomáticos que trabajan para las Naciones Unidas.

\footnotetext{
${ }^{9}$ Vilariño, Eduardo (2007): Curso de Derecho Diplomático y Consular, $3^{\circ}$ Ed., Madrid, Tecnos, p. 79.
} 
c) Es llevada a cabo por los órganos plenamente representativos ya que no todo el mundo puede ostentar la representación de un sujeto de derecho internacional.

d) La principal función de la diplomacia hoy es la negociación y alcanzar acuerdos a través del diálogo tal y como ha señalado reiteradamente Adam Watson.

e) Por último, es imprescindible que las relaciones diplomáticas se desarrollen en un entorno pacífico. De hecho, a través de la diplomacia se pretende mejorar las relaciones entre los distintos actores que componen la sociedad internacional. Como curiosidad, se puede añadir que una negativa reitera al establecimiento de relaciones diplomáticas bien podría ser considerada como un acto contrario al espíritu y a los principios de la Carta de las Naciones Unidas.

\section{El Servicio Diplomático Español}

Si bien encontramos muchas referencias a la diplomacia en el mundo antiguo, como pueda ser las alusiones en el Artha-Sastra en la India o los colegios fetiales en Roma, la diplomacia moderna es mucho más cercana en el tiempo. En España podemos fechar las primeras andanzas de nuestro Servicio Exterior con los Reyes Católicos, cuando las misiones especiales se convierten en misiones permanentes. Fue Fernando el Católico quien acreditó a los primeros embajadores permanentes en Londres, Santa Sede, Borgoña o Lisboa. Sin embargo, no fue hasta 1778 cuando el ministro de Carlos III Floridablanca nacionalizó y profesionalizó el Servicio Exterior de España.

La primera organización profesional del servicio diplomático español no llegó hasta 1844 cuando el entonces Ministro de Estado ${ }^{10}$ González Bravo ordenó mediante un Real Decreto la carrera diplomática española. Siguiendo el modelo de diplomacia francesa, se aprobó un derecho por el cual el ingreso en la carrera diplomática estaba basado en los méritos y en la capacidad con altos niveles de exigencia. España optaba por lo que Harold

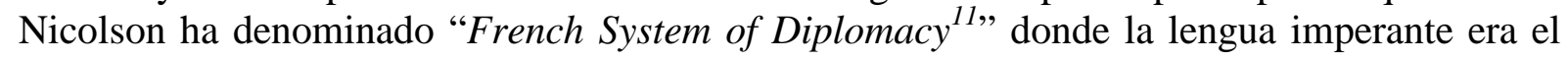
francés, el protocolo era muy importante y la profesionalización esencial. En ocasiones los sistemas diplomáticos que seguían esta orientación eran dominados por la aristocracia ${ }^{12}$.

El decreto de 1844 establecía una serie de categorías dentro del Servicio Exterior de España que tenía un reconocimiento internacional. Así la carrera diplomática se componía de:

a) Embajadores Ordinarios.

b) Embajadores Extraordinarios

c) Ministros Plenipotenciarios

d) Ministros Residentes

e) Encargados de Negocios

\footnotetext{
${ }^{10}$ Esta era la denominación utilizada para los Ministros de Asuntos Exteriores.

${ }^{11}$ Nicholson, Harald (1954): The Evolution of the Diplomatic Method, London, Constable.

${ }^{12}$ Berridge, op. cit., p. 108.
} 
f) Secretarios de Legación de primera, segunda y tercera clase ${ }^{13}$.

g) Agregados Diplomáticos de Planta

h) Agregados Diplomáticos sin sueldo.

En 1851 se modificó la regulación de la carrera diplomática mediante la aprobación de otro decreto y la eliminación de los embajadores ordinarios así como de los agregados de planta. Además se establecieron las siguientes categorías:

a) Embajador

b) Ministro Plenipotenciario de Primera y Segunda Clase

c) Encargados de Negocios

d) Secretarios de Primera, Segunda y Tercera Clase

La ausencia de claridad respecto de la obligatoriedad de aprobar un examen, se hablaba de la necesidad de haber superado un número de asignaturas con la calificación de sobresaliente. Esta cláusula permitía que el gobierno tuviera un importante papel en el nombramiento de embajadores, lo que hoy conocemos como embajadores políticos. El verdadero cambio que permitió que el Servicio Exterior diera un salto de calidad fue la aprobación del Estatuto de Bravo Murillo (1852).

En 1883 se culminó la modernización del servicio diplomático español con la aprobación de la Ley Orgánica de las Carreras Diplomáticas, Consular y de Intérpretes. Dicha ley establecía la oposición -grand concours- como medio de ingreso en la Carrera Diplomática aunque dejaba abierta la puerta contratar agregados o a nombrar embajadores no de carrera cuando concurrieran circunstancias especiales.

En lo que a los ascensos se refiere se mezclaban dos criterios: el de antigüedad y el del mérito por lo que de cada tres plazas de ascenso dos se cubrirían de acuerdo a la antigüedad y la otra conforme a los méritos. Lejos quedaban otros métodos como "up-or-out" del Foreign Service americano.

Otro elemento característico del servicio exterior y que lo diferenciaba de otros cuerpos era la no retribución del servicio. Tras el ingreso en el cuerpo diplomático no se asignaba ninguna remuneración, al menos en los primeros años, lo que hacia de la Carrera Diplomática un cuerpo elitista reservado a hombres de fortuna o a la aristocracia. Este elemento también se daba en otros servicios diplomáticos y de hecho entre cónsules se mantuvo durante muchos años. En ocasiones los cónsules eran nacionales de fortuna que tenían sus negocios, sobre todo en los puertos, que no obtenían ninguna contraprestación económica pero que se beneficiaban de un cierto estatus que les permitía desarrollar su negocio en mejores condiciones.

\footnotetext{
${ }^{13}$ En la actualidad aunque la Convención de Viena reconoce legaciones de segundo tipo, no se usa. Las de tercera clase solo se usan cuando se produce una crisis y el Estado Acreditante opta por reducir el rango del Jefe de Misión.
} 
En 1928, con la Dictadura de Primo de Rivera llegó la unificación de las carreras diplomática y consular. Este hecho, no sólo se dio en España también en otros lugares como los Estados Unidos se decidió unificar los dos cuerpos. Si bien es cierto que los cónsules acogieron esta decisión con alegría, los diplomáticos no lo vieron con buenos ojos.

En 1932 se introdujeron de forma oficial el estudio de dos lenguas: la inglesa y la francesa. Además se incluyeron una serie de medidas que pretendían acabar con el sistema de recomendaciones haciendo de la carrera diplomática un cuerpo más capacitado y sobre todo más justo. La Roger Act aprobada por el Congreso norteamericano tuvo cierta influencia en el Servicio Exterior de España buscando la profesionalización y la excelencia de sus miembros. Sin embargo, los avatares de la Segunda República también afectaron a la Carrera Diplomática con la aprobación de un decreto en el que se afirmaba que "estaba abierta a los jóvenes de toda clase social ${ }^{14,}$

La Guerra Civil y posterior dictadura del General Franco supuso alguna depuración en el servicio. No obstante, un $84 \%$ de los diplomáticos recibieron el calificativo de "admitido" tras la victoria del bando denominado nacional. Como será comentado con posterioridad, el Gobierno del Presidente Zapatero ha aprobado algunas disposiciones legales para restaurar la memoria y el honor de aquellos que fueron separados del servicio. Sin embargo, el Régimen de Franco continuó con las reformas profesionales emprendidas durante la Segunda República.

Los que sí fueron separados del servicio y recuperados por el Gobierno del Presidente Aznar, fueron un grupo de diplomáticos españoles que salvaron de una muerte segura a miles de judíos que se encontraban en territorios controlados por el Tercer Reich. Caben ser destacados entre otros: Eduardo Propper de Callejón, Bernardo Rolland de Miota, Sebastián Romero, Julio Palencia, José Ruiz Santaella, José Rojas y Ángel Sanz Briz ${ }^{15}$.

Uno de los puntos más importantes para la diplomacia española fue la creación de la Escuela Diplomática en 1942. Al igual que se creó el Foreign Service Institute en los Estados Unidos o École diplomatique et consulaire en Francia, la Escuela Diplomática nació para formar a los diplomáticos que acababan de aprobar su oposición y que se disponían a afrontar su primer destino ${ }^{16}$. Hoy casi 70 años después continúa siendo el principal centro de formación de los diplomáticos.

Desde su creación hasta hoy, se han ido aprobando distintas disposiciones legales que han ido regulando y modificando las condiciones de acceso a la Carrera Diplomática así como el papel de la Escuela Diplomática. Sin embargo, los resultados aunque han sido positivos no son suficientes. Recientemente se comenzó a pensar de forma más clara en la necesidad de reformar el Servicio Exterior de España ya que por un lado lo obsoleto del sistema y por el otro la redimensión de España como potencia requería una reforma del instrumento de la implementación de la política exterior.

En 1986 con la entrada de España en las entonces Comunidades Europeas, se elaboró un informe denominado Libro Blanco Sobre la Administración Exterior del Estado que apuntaba

\footnotetext{
${ }^{14}$ Pereira, op. cit., p. 238.

${ }^{15}$ Sobre este tema ver Marquina, Antonio y Ospina, Gloria (1986): España y los judios en el siglo XX, Madrid, Espasa Calpe.

${ }^{16}$ Previamente existió lo que se conoció como el Instituto de Enseñanzas de las Carreras Diplomática y Consular y Centro de Estudios Marroquíes.
} 
importantes carencias en nuestro Servicio Exterior. Sin embargo, debido a la situación de precariedad económica dichas reformas no se llevaron a cabo.

En 2004, el PSOE en su programa electoral para las elecciones generales llevaba una propuesta que no era otra cosa que la reforma del Servicio Exterior Español ${ }^{17}$. En junio de 2004, tras la victoria electoral, se formó la Comisión Interministerial para la Reforma Integral del Servicio Exterior ${ }^{18}$ con la función de llevar a cabo los cambios necesarios para la modernización del Cuerpo Diplomático. La Comisión estaba presidida por el Embajador en Misión Especial para la Reforma del Sector Exterior D. Melitón Cardona. Junto a él dos representantes del Ministerio de Economía y Hacienda más un representante de cada uno de los ministerios con la excepción del Ministerio de Vivienda que no estaba representado por nadie. También contó con un Abogado del Estado ${ }^{19}$.

En octubre de 2004 el recién nombrado Ministro de Asuntos Exteriores se dirigió a las embajadores españoles en una alocución en la que marcaba los puntos clave de la reforma que acaban de iniciar. Según el titular de exteriores se debía "abandonar el patrón jerárquico y rígido convirtiéndose en un proveedor de servicios originales al gobierno, al Parlamento, a las administraciones públicas centrales y autonómicas, a las empresas, a la pluralidad de las organizaciones y asociaciones que integran la sociedad civil y al ciudadano en particular ${ }^{20,}$

La Comisión trabajó durante 12 meses para identificar dos misiones fundamentales. En primer lugar identificar las carencias propias de nuestro servicia exterior español y en segundo lugar que acciones se podrían tomar para solucionarlas. El informe se dividió en tres partes:

1) La primera parte del informe trata de analizar los cambios acaecidos en la Sociedad Internacional que hacen necesaria la reforma del Servicio Exterior. La idea central es que los cambios que está sufriendo el sistema internacional no pueden ser afrontados con este Servicio Exterior de España. Por ello, aunque no se cite de forma explícita se debería pasar de lo que se ha conocido como Vieja Diplomacia o tradicional hacia una Nueva Diplomacia o estructural.

2) La Segunda parte del Informe está centrada en un análisis y posterior evaluación de la situación del Servicio Exterior y adecuación a las nuevas realidades. Quizás una de las críticas que se hace al servicio diplomático es la no hacer una evaluación adecuada de las realidades. Sin embargo, este aspecto no debería corresponder tanto a los propios diplomáticos sino al conjunto de formuladores de la política exterior incluyendo en esto a distintos sectores de la sociedad civil.

3) La tercera y última parte del informe está centrado en las medidas concretas de carácter normativo, organizativo y presupuestario, necesarias para llevar a cabo las reformas. Aquí llegamos a uno de los puntos clave. A pesar de que desde 1996 se viene trabajando en el incremento de las partidas presupuestarias destinadas al Ministerio de Asuntos Exteriores, sigue siendo uno de los departamentos del gobierno español con menor dotación presupuestaria. Además no sólo está en desventaja en España sino que el MAEC hoy es uno de los Ministerios de Asuntos Exteriores con menos recursos de nuestra zona.

\footnotetext{
${ }^{17}$ Cardona, Melitón: "La reforma del servicio exterior español”, $A B C, 2$ de enero de 2008.

${ }^{18}$ Comisión creada por Acuerdo de Consejo de Ministros de 24 junio de 2004.

${ }^{19}$ Ver BOE núm. 154 de 26 de junio de 2004.

${ }^{20}$ Riordan op. cit., p. 5.
} 
En junio de 2005 el informe final fue remitido al gobierno avalado por los representantes de los 15 ministerios implicados que inspiró al ejecutivo para la reforma. El Gobierno asumió la totalidad de las recomendaciones elaboradas por "el consejo de sabios" mediante Acuerdo de Consejo de Ministros fechado el 1 de septiembre de 2006.

\section{La Reforma de 2006}

Tras la aprobación de las recomendaciones establecidas por la Comisión en 2005 se inició un plan para reformar el servicio exterior ${ }^{21}$ teniendo en cuenta las necesidades establecidas, los recursos disponibles y los objetivos marcados.

Uno de los principales aspectos de la reforma era la justificación de la misma. Tres fueron los aspectos que se establecieron para llevar a cabo dicha reforma:

1. España aspiraba a mantenerse entre las 8 economías más pujantes del planeta y para ello era fundamental mantener y potenciar nuestro sector exterior. Se calculaba que alrededor de 21 millones de puestos de trabajo dependían del exterior, sin contar con los 1.5 millones de españoles que viven en el extranjero. Además se calcula que alrededor de 10 millones de españoles viajan al menos una vez al año al extranjero por lo que es importante mantener una importante red de consulados para asistirlos ${ }^{22}$.

2. España posee una de las lenguas más habladas del mundo y por ello, para mantener ese activo era necesario tener una presencia acorde a dicho patrimonio. De hecho, se pretendía utilizar y potenciar la imagen internacional de la "Marca España" como activo para mejorar nuestra presencia global. En esta línea se habían desarrollado iniciativas interesantes como la potenciación del Instituto Cervantes, creado en 1991, y potenciado a comienzos de este siglo.

3. Hacerse eco de una "supuesta" demanda social para encaminar la política exterior española hacia unos fines solidarios que eran reclamados por la Sociedad Española. Este particular ha sido una constante durante las dos legislaturas tal y como demuestra el cambio de nombre del Ministerio de Asuntos Exteriores que desde 2004 incorporó la palabra Cooperación. Sin embargo, sin considerar un error dicha aportación si que debería usarse más esta cuestión para la consecución de los fines de la política exterior de España.

Lo que se aprecia con el asunto de la reforma es una cierta confusión entre diplomacia y política exterior ya que de lo que se había hablado tanto en el programa electoral del PSOE como en el discurso de Miguel Ángel Moratinos a los embajadores está más centrado en la política exterior que en el servicio exterior. No obstante la reforma se hace necesaria aunque no es menos cierto que existía la percepción de que el Servicio Exterior Español funcionaba y

\footnotetext{
${ }^{21}$ Ministerio de la Presidencia "Informe sobre la Reforma del Servicio Exterior Español” 20 junio de 2005.

${ }^{22}$ Según el MAEC, en la actualidad España cuenta con unas 200 Oficinas y Secciones Consulares y más de 500 Consulados y Consulados Honorarios.
} 
funciona bien $^{23}$ y en buena medida se debe al fantástico equipo humano que forman nuestro servicio, reconocido dentro pero sobre todo fuera de nuestras fronteras.

Basándose en estas necesidades la Comisión Interministerial que desarrolló el informe propuso una reforma del servicio exterior basada en seis ejes:
1. Coordinación
2. Modernización
3. Profesionalidad
4. Especialización
5. Homogeneidad
6. Simplificación de procedimientos.

que en total generaron más de 60 propuestas ya más concretas entre las que pueden destacarse las siguientes:

a) Superar la escasa planificación de la acción exterior planteando Planes Conjuntos de Acción Exterior que contemplen objetivos a corto, medio y largo plazo. A esto quizás habría que añadir que se deben dar pactos políticos para convertir a España en un actor predecible y con una identidad propia a nivel internacional. Así, se facilitaría el trabajo de los diplomáticos y se eliminarían los "embajadores políticos" que llegan con un trabajo determinado a realizar.

b) Avanzar en la coordinación potenciando el Consejo de Política Exterior. De hecho se ha aprobado un Real Decreto (1389/2007 de 29 de octubre) donde se incrementan los poderes del Consejo de Política Exterior elevando el rango de sus recomendaciones

c) Incrementar los medios destinados al personal pero no sólo en cuanto a cantidad sino también en calidad ya que se plantea la necesidad de especialización geográfica y temática. A pesar de algunos cambios establecidos, la Escuela Diplomática debe convertirse en el principal centro de pensamiento sobre Diplomacia incluyendo la contratación de investigadores que abran las líneas fundamentales en este campo al tiempo que estén en contacto con los principales think-tanks sobre este tema.

d) Necesidad de dotarse de instrumentos legales que regule el Servicio Exterior como una rama singular de la Administración reconociendo así sus especiales características. Al igual que ocurre en el Foreign Service se separó del Senior Act hay una necesidad de diferenciar este cuerpo de características especiales del resto de la administración. El Foreign Service tiene características propias en cuanto a

\footnotetext{
23 "En términos generales, se puede afirmar que los servicios españoles en el exterior funcionan razonablemente bien": Espósito, Carlos: "Reflexiones sobre la reforma del Servicio Exterior Español" FRIDE-Documento de Trabajo (Febrero 2005), p. 3.
} 
salarios, destinos, prerrogativas etc... derivadas todas de hechos tales como que el diplomático se pasa dos tercios de su vida fuera de su país ${ }^{24}$.

\section{Conclusiones y recomendaciones}

Este ambicioso -quizás demasiado- a la par que necesario programa por el que se trataba de dotar a España de un servicio exterior adecuado a su peso internacional debe ser evaluado de forma adecuada para establecer que puntos han sido alcanzados y cuáles no. Del mismo modo se pueden incluir algunas otras ideas que a mi juicio deberían formar parte de la citada reforma.

1) La primera crítica es que se confunden niveles ya que algunas de las recomendaciones no están tan destinado a la diplomacia o a la institución consular como a la política exterior. Esta crítica es especialmente aplicable tanto a la propia coordinación como sobre todo a la planificación. En ambos casos no estaríamos hablando tanto de la diplomacia sino del policy-making a nivel de Ministerio de Asuntos Exteriores.

De hecho, si nos centramos en la coordinación apreciamos que dicho problema se da tanto a nivel de ministerios, como a nivel de departamentos tanto en la propia misión como a nivel de los distintos entes administrativos. Por ello uno de los principales problemas señalados por el informe de 2005 está hoy por solventar.

2) El segundo de los puntos que merece ser criticado es el eje que se centra en la profesionalidad. Este aspecto choca frontalmente con el abuso del nombramiento de embajadores de carácter político ya que los dos gobiernos presididos por el Presidente Rodríguez Zapatero tienen el record de ser el gobierno democrático que más uso ha hecho de esta herramienta que choca frontalmente con la profesionalidad del servicio diplomático.

\footnotetext{
${ }^{24}$ Priego, Alberto: "El Servicio Diplomático Norteamericano: El Foreign Service” Tribuna Norteamericana, no 6 (Julio de 2011).
} 
GRÁFICO 3: Nombramiento de Embajadores políticos en la democracia

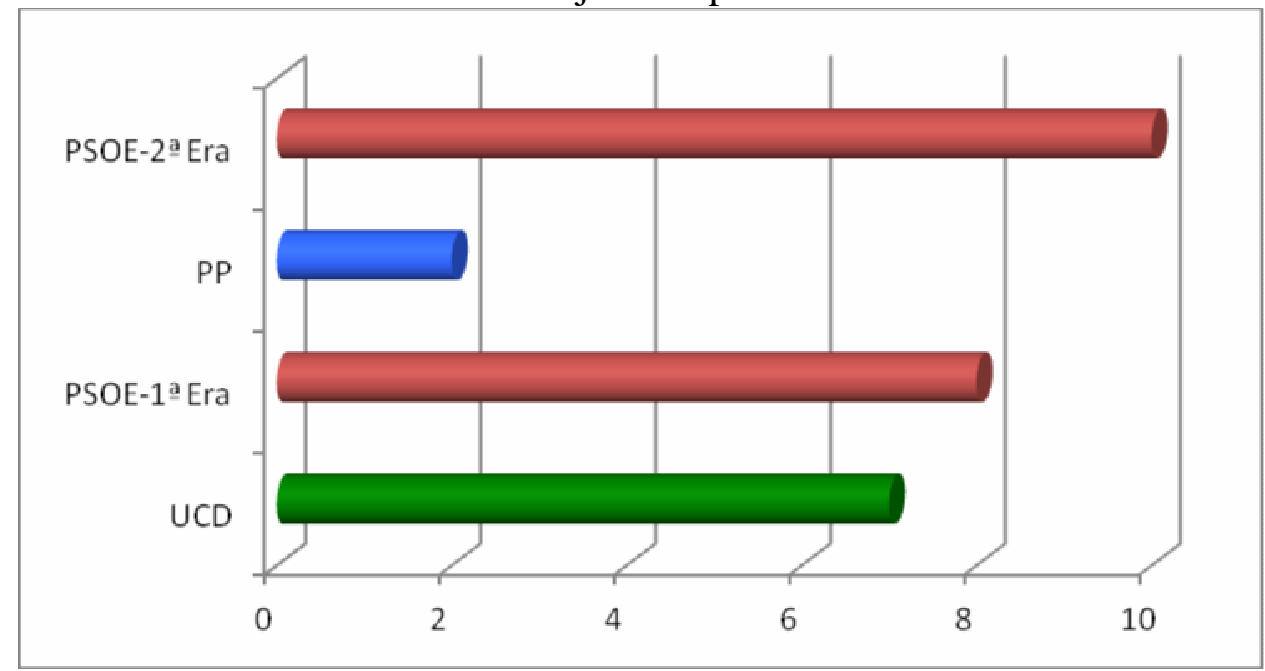

FUENTE: Pereira, Juan Carlos (Coord.) (2010): La Política Exterior de España. De 1800 hasta hoy. Ed. Ariel. Madrid pp. 248 y siguientes.

Llama la atención que haya sido el gobierno que más uso ha hecho de este sistema de nombramiento de embajadores sobre todo porque contrasta con este eje de la reforma del sector exterior señalado por el informe de 2005. Si bien es cierto que se ha intentado copiar el sistema norteamericano donde prima la profesionalización y la especialización -a pesar de algunos nombramientos políticos- en general el Foreign Service no se apoya tanto en personal ajeno al Departamento de Estado. Algunos autores como Ignacio Molina incluso hablaban de la "despartidización" en el Servicio Exterior Español durante el periodo Aznar ${ }^{25}$.

Además del incremento en cantidad de los embajadores políticos también hay que decir que estos han ocupado misiones diplomáticas de mayor importancia para España. Si bien el ejecutivo anterior nombró este tipo de figuras en misiones de un menor perfil como la de la OCDE, el ejecutivo de Rodríguez Zapatero ha copado con diplomáticos no profesionales misiones tan importantes como Turquía ${ }^{26}$, Argentina ${ }^{27}$ o Santa Sede ${ }^{28}$.

3) Algunos de los elementos más negativos ha sido la ideologización del servicio diplomático y la aplicación de la memoria histórica a dicho cuerpo con la aprobación ${ }^{29}$ de la Ley 52/2007. Llama la atención que se haya esperado a 2007 para "restaurar el honor" de los diplomáticos perseguidos por el Régimen de Franco. Por su parte el Partido Popular ya llevó a cabo una acción similar, pero con aquellos diplomáticos que salvaron a población judía de morir en campos de concentración.

\footnotetext{
${ }^{25}$ Molina, Ignacio y Rodrigo, Fernando: "Las Transformaciones organizativas de la política exterior española", Revista de Estudios Políticos, no. 117 (Julio-Septiembre de 2002), p. 187.

${ }^{26}$ Joan Clos ex Alcalde de Barcelona y ex Ministro de Industria quien además está acreditado en Azerbaiyán en régimen de acreditación múltiple.

${ }^{27}$ Rafael Estrella quien ha sido diputado del Partido Socialista Obrero Español.

${ }^{28}$ Francisco Vázquez, ex-alcalde socialista de A Coruña.

${ }^{29}$ Ley 52/2007 de 26 de noviembre.
} 
4) A pesar de haber sido considerada como una de las prioridades para España, la reforma del sector exterior ha sufrido graves recortes en el año 2011. Podemos decir que si bien los recortes se esperaban quizás una mejor distribución del gasto favorecería la consecución de los logros establecidos en el informe de 2005 como por ejemplo la proporción que aporta exteriores a la cooperación al desarrollo. Si bien mientras que en 2004 suponía un 19.8\% del total, en 2011 será el 48.9\%. Quizás habría que encontrar otras soluciones antes que recortar nuestras propias necesidades ${ }^{30}$ como puedan ser la no apertura de nuevas misiones o el anuncio de supresión, finalmente no consumado, de la importante Secretaría de Estado para la UE.

La gran totalidad de los grupos políticos criticaron la "frustrada reforma" del servicio exterior así como la falta de dotación presupuestaria de dicha reforma. En definitiva la reforma ha estado mal planteada y poco dotada de medios especialmente teniendo en cuenta lo ambicioso del proyecto original ${ }^{31}$.

5) Otro de los elementos quizás serían de gran utilidad sería incorporar a diplomáticos a otros departamentos tal y como hace el Foreign Service norteamericano que cuenta con personal en otras agencias como USAID, Department of Defence o Department of Agriculture. Con este punto podría subsanarse un aspecto que se ha detectado en el informe como algo a mejorar, la coordinación de la política exterior a través de los diferentes niveles de gobierno. Sin romper el principio de unidad de acción garantizado por la Constitución del 78 si que se hace necesaria una coordinación de la política exterior, tarea esta que corresponde a los órganos centrales, es decir al gobierno central.

Al mismo tiempo, y al haber logrado la creación de un Servicio Exterior de Acción Exterior en la UE también debemos tener en cuenta que nuestro servicio exterior tiene una nueva dimensión y que será un ente más con quien coordinar nuestra política exterior y una ayuda a la hora de ejecutar ésta ${ }^{32}$. Por lo tanto sin perder la referencia de los órganos centrales como centro de la política exterior se deben tener en cuenta a otros niveles para no duplicar esfuerzos.

6) Por otro lado, también sería importante clarificar si queremos y estamos dispuestos a cambiar verdaderamente el modelo de diplomacia. Tal y como decía, el entonces Ministro de Asuntos Exteriores Miguel Moratinos nuestro modelo quizás es demasiado jerárquico y basado en la diplomacia tradicional y se debería impulsar su cambio. Pudiendo ser esto cierto quizás, también sería necesario que los dos grandes partidos con posibilidades de gobernar pudieran pactar el modelo para evitar así continuos cambios provocados por la alternancia de partidos Por supuesto que deben continuarse interesantes iniciativas como Casa Árabe, Casa Asia, Instituto Cervantes o

\footnotetext{
${ }^{30}$ Ver "Presupuestos. El Congreso convalida la partida destinada a Exteriores para 2011" disponible en http://www.periodistadigital.com/mundo/africa/2010/11/16/presupuestos-el-congreso-convalida-la-partidadestinada-a-exteriores-para-2011.shtml.

31 "Los grupos parlamentarios de PP, IU-ICV y CiU reprocharon hoy al Gobierno que no haya reformado el servicio exterior, como propuso en su día, y que tampoco el proyecto de Presupuestos Generales del Estado (PGE) para 2008 dedique una partida para acometer esta iniciativa”, Europa Press, 18 de Noviembre de 2009.

${ }^{32}$ Priego, Alberto: "El Servicio Europeo de Acción Exterior: ¿una revolución para el proceso de integración y para la diplomacia?" 95/2011, 25 de mayo de 2011. Disponible en

http://www.realinstitutoelcano.org/wps/wcm/connect/47d0188046f62442aecbbe00526b8882/ARI952011_Priego_Servicio_Europeo_Accion_Exterior_diplomacia.pdf?MOD=AJPERES\&CACHEID=47d0188046f 62442aecbbe00526b8882.
} 
la Academia de Roma pero siendo conscientes que nos dirigimos a un modelo de Nueva Diplomacia o Diplomacia Estructural con lo todo lo que ello conlleva.

7) Uno de los aspectos menos tratado en la reforma es el sistema de los ascensos. De nuevo el sistema americano puede mostrarse como una referencia en dos direcciones. En primer lugar, con el sistema de promoción up-or-out copiado del ejército en el que si en un determinado periodo de tiempo no se logran los ascensos deseados el diplomático debe abandonar el cuerpo. En segundo logar introduciendo la categoría de Senior Officer que no logra todo el que entra en el Foreign Service. Con estas dos simples medidas se lograría una mayor motivación del personal diplomático ya que se evitarías las "recomendaciones" y los alineamientos políticos.

8) En esta misma línea el sistema de incorporación también necesita ser reformado y también el Foreign Service presenta opciones interesantes. El famoso Foreign Service Officer Test (FSOT) es un examen menos solemne que nuestra oposición a cuerpo diplomático pero donde se tienen en cuenta las habilidades y conocimientos del candidato. De hecho es el Departamento de Estado el que viaja por todo Estado Unidos captando candidatos cuyos primeros exámenes pueden ser realizados desde su propia ciudad de residencia. También el Servicio Europeo de Acción Exterior presenta importantes novedades como la composición mixta de sus miembros: $33 \%$ son funcionarios de la UE, 33\% diplomáticos en comisión de servicios y el resto convocatoria pública abierta a cualquier ciudadano que cumpla los criterios de la convocatoria. Tanto en un caso como en el otro la mayor diversidad de candidato permite una mejor adaptación a las nuevas realidades que se señalaban en las primeras páginas del informe de la Comisión encargada de la reforma.

9) También otro de los elementos que deberían haberse tenido en cuenta son las nuevas tecnologías, algo de crucial importancia para la diplomacia hoy. Funciones tan importantes como la información, el envío de cables, o el feed-back con su ministerio es más sencillo. Sin embargo se debe hacer un uso mayor de las nuevas tecnologías para favorecer el trabajo de los diplomáticos ${ }^{33}$. Si en la diplomacia las tecnologías son de crucial importancia, en los asuntos consulares es aún más importante. El uso de teléfonos móviles permite un mayor contacto entre el funcionario consular y el ciudadano. Sin embargo, existen el uso de teléfonos móviles puede quedar anulado en situaciones de emergencia como un atentado terrorista. No obstante, el uso de otras tecnologías como el VoIP facility -por ejemplo Skype o Tango- permitió a la High Commission de Malta poderse comunicar con sus ciudadanos durante las horas posteriores a los atentados del 7-J. Este tipo de recomendaciones también se echan en falta en la reforma de nuestro servicio exterior.

10) Según el propio Ministerio de Asuntos Exteriores y Cooperación en la actualidad hay 1.500.000 de españoles viviendo en el exterior. Este hecho es visto habitualmente como un "problema" y no como una oportunidad para la promoción de la "Marca España". A pesar de que no somos capaces de asumir la producción de trabajadores de alta cualificación y que una buena parte tiene que cruzar la frontera para ganarse la vida, el perfil del español viviendo fuera ha variado mucho. Ya no estamos ante el ciudadano sin formación que tiene una ocupación de baja formación. Hoy nuestros españoles son parte de nuestra diplomacia ya que dan una buena imagen de nuestro

\footnotetext{
33 Radunovic, Vladimir: "The Role of Information and Communication Technologies in Diplomacy and Diplomatic Service”, Contemporary Diplomacy, (Mayo 2010) pp. 44-46.
} 
país y por ello deberían ser cuidados mejor por nuestro gobierno. Por ejemplo Italia posee un ministerio de la diáspora y, sin llegar a tanto, se les debería conceder más importancia $^{34}$.

11) Otro de los aspectos que tampoco han sido tenido en cuenta es el tema de la seguridad de las embajadas. Ser diplomático es una profesión cada vez más peligrosa. Por ello, los Estados Unidos han creado cuerpos nuevos para la protección de sus nacionales, como los CORD, y están trabajando en el rediseño de los edificios que acogen las misiones. Muchas de las principales misiones han sido reubicadas en zonas menos peligrosas y los edificios adaptados a las nuevas condiciones de seguridad. Este es un tema tabú en España que debe ser afrontado mediante un debate serio y despolitizado.

Otros servicios diplomáticos también han iniciados procesos de reforma como el Foreign Service. Sin embargo, las líneas maestras adoptadas por Departamento de Estado deberían ser una referencia para nuestro Ministerio de Asuntos Exteriores y Cooperación. Tres son los documentos elaborados por Washington para la reforma de su servicio diplomático: el Diplomatic Readniness Initiative (DRI) de 2001, la orden presidencial NSDP 44 de 2005 y el programa Diplomacy 3.0 de $2008^{35}$. En ningún caso se han tenido en cuenta para la reforma de nuestro servicio y como se puede deducir de la fecha al menos dos de ellos son anteriores a la publicación del informe de 2005.

También el Foreign Office está en un proceso de reforma. Entre los principales temas está la reducción del gasto y el apoyo a las empresas británicas en el exterior. El mayor pragmatismo así como el apoyo a sus nacionales en el exterior ha sido una de las líneas caracteristicas de la política llevada a cabo por Whitehall. Por ello a pesar de los cambios propuestos para mejorar y modernizer el servicio estas particularidades no deben ser modificadas ${ }^{36}$ ni mucho menos eliminadas.

A modo de conclusión final debemos decir que la introducción del debate de la reforma del servicio exterior e incluso la elaboración del informe debe ser evaluado de forma positiva, aunque el resultado real de dicha reforma esté lejos de las expectativas creadas con la iniciativa de 2005. La crisis económica, los obstáculos ideológicos y algunas barreras históricas han impedido llevar a cabo una verdadera reforma del Servicio Exterior Español.

\footnotetext{
${ }^{34}$ Bátora, J. (2005): Public diplomacy in small and medium-sized states: Norway and Canada, Clingendael, Netherlands Institute of International

Relations, p. 55.

${ }^{35}$ Priego, Alberto: "El Servicio Diplomático Norteamericano...”, op. cit., p. 12.

36 "Time to reform the failing Foreign Office", Daily Mail, 6 de enero de 2011.
} 